OPEN ACCESS

Fabrication of Three-Dimensional (3D) Copper/Carbon Nanotube Composite Film by One-Step Electrodeposition

To cite this article: Susumu Arai et al 2016 J. Electrochem. Soc. 163 D774

View the article online for updates and enhancements. 


\title{
Fabrication of Three-Dimensional (3D) Copper/Carbon Nanotube Composite Film by One-Step Electrodeposition
}

\author{
Susumu Arai,*,z Masaya Ozawa, and Masahiro Shimizu* \\ Department of Chemistry and Material Engineering, Faculty of Engineering, Shinshu University, Nagano-shi, Nagano \\ 380-8553, Japan
}

\begin{abstract}
A three-dimensional (3D) composite film containing copper nanostructures and carbon nanotubes (3DC/CNT composite film) was fabricated by one-step electrodeposition. The 3DC/CNT composite film was formed under galvanostatic conditions using a copper sulfate bath containing CNTs and polyacrylic acid which acts as both a 3DC-forming and a CNT-dispersing agent. The composite film consists of thin copper sheets with thicknesses of ca. 70-80 nm and CNTs, with large interior spaces between sheets. The CNTs were homogeneously distributed inside the composite film and were fixed by the copper sheets where CNTs pierce the copper sheets. The CNT content in the composite films increased with the CNT concentration of the plating bath. The 3DC film without CNTs did not maintain its 3D spaces when the film thickness was increased due to insufficient structural strength, whereas the 3DC/CNT composite film maintained the 3D spaces despite an increase in film thickness, which suggests that the CNTs reinforce the film to maintain the $3 \mathrm{D}$ spaces.

(C) The Author(s) 2016. Published by ECS. This is an open access article distributed under the terms of the Creative Commons Attribution 4.0 License (CC BY, http://creativecommons.org/licenses/by/4.0/), which permits unrestricted reuse of the work in any medium, provided the original work is properly cited. [DOI: 10.1149/2.0601614jes] All rights reserved.

(cc) BY
\end{abstract}

Manuscript submitted August 30, 2016; revised manuscript received October 13, 2016. Published November 4, 2016.

Three-dimensional (3D) nanostructured metal architectures typically exhibit large specific surface areas and high electrical conductivity; therefore, their application as electrodes in functional devices such as supercapacitors, ${ }^{1}$ fuel cells ${ }^{2}$ and batteries ${ }^{3}$ have been widely researched. For these potential applications, the use of 3D copper nanostructured architectures ${ }^{4-8}$ is most attractive. However, although 3D copper nanostructured architectures are very effective to improve electrode functions, their manufacture requires many steps and is therefore complex. Our group has previously developed a very straightforward method for the fabrication of 3D copper nanostructured architectures by electrodeposition, in which an organic additive is simply added to an electrodeposition bath. ${ }^{9}$ This process for the preparation of 3D copper nanostructured architecture films by onestep electrodeposition (the resulting products henceforth being designated as 3DC1 film) is expected to be used as a practical method for the fabrication of various components, such as current collectors for tin-based lithium-ion battery anodes. ${ }^{10}$ However, the 3DC1 film consists of very thin (30-50 nm) copper sheets; therefore, its structural strength is low, which results in deformation of the 3D structure when a slight physical force is applied.

In contrast, carbon nanotubes $(\mathrm{CNTs})^{11,12}$ have excellent mechanical characteristics, ${ }^{13-15}$ such as high tensile strength and high elastic modulus, in addition to high thermal ${ }^{16-18}$ and electrical conductivity. ${ }^{19,20}$ Research into CNT composites such as metal/CNT composites has thus been actively pursued. ${ }^{21,22} \mathrm{~A} 3 \mathrm{D}$-copper nanostructured architecture containing CNTs (3DC/CNT composite) is thus considered to be an attractive copper-based $3 \mathrm{D}$ composite material that would have sufficient structural strength due to the reinforcement effect of the CNTs. If a 3DC/CNT composite could be fabricated by a simple method, then the 3DC/CNT composite materials would be expected to be used for a wider range of practical applications. We have also examined the electrodeposition of metal/CNT composite films and reported the fabrication and characteristics of dense $\mathrm{Cu} / \mathrm{CNT}$ composite films. ${ }^{23}$ Consequently, a combination of the 3DC1 technology and the $\mathrm{Cu} / \mathrm{CNT}$ composite plating technology is expected to produce a practical 3DC/CNT composite film material.

In this study, the fabrication of 3DC/CNT composite films by one-step electrodeposition and the resulting microstructure of the 3DC/CNT composite films were investigated.

\section{Experimental}

Commercially available vapor-grown multi-walled CNTs (Showa Denko Co. Ltd.) were formed via catalyst-assisted chemical vapor deposition (CVD) ${ }^{24}$ and heat treated at $2800^{\circ} \mathrm{C}$ in an argon gas atmosphere for $30 \mathrm{~min}$. The CNTs were typically $100-150 \mathrm{~nm}$ in diameter and $10 \mu \mathrm{m}$ long. An acidic copper sulfate bath composed of $0.85 \mathrm{M}$ $\mathrm{CuSO}_{4} \cdot 5 \mathrm{H}_{2} \mathrm{O}$ and $0.55 \mathrm{M} \mathrm{H}_{2} \mathrm{SO}_{4}$ was used as the base plating bath. CNTs and polyacrylic acid (mean molecular weight 5000; PA-5000), which should act not only as a $3 \mathrm{DC}$ forming agent ${ }^{11}$ but also as a dispersant of CNTs, ${ }^{25,26}$ were added to the base plating bath to prepare a 3DC/CNT composite plating bath. The concentration of PA-5000 was consistently $3 \times 10^{-4} \mathrm{M}$ throughout this study. A 3DC1 plating bath (base plating bath $+3 \times 10^{-4}$ M PA-5000) without CNTs was also prepared for comparison.

The dispersibility of CNTs in the plating baths was evaluated using a laser diffraction particle size analyzer (SALD-7000, Shimadzu Seisakusho). The composite plating baths containing 2-10 $\mathrm{g} \mathrm{dm}^{-3}$ CNTs were diluted with pure water to prepare a suitable particle concentration for dispersibility measurements. The zeta potentials of the CNTs in the plating baths were also measured using an electrophoresis type zeta potential measurement system (Model 502, Nihon Rufuto), where the composite plating baths were diluted with pure water to prepare suitable measurement conditions. The plating solutions are aqueous solutions; therefore, the zeta potentials were calculated using the Smoluchowski equation:

$$
\mu=\frac{\varepsilon_{0} \varepsilon_{\mathrm{r}} \zeta}{\eta}
$$

where $\mu$ is the electrophoretic mobility, $\varepsilon_{0}$ is the relative dielectric constant of water, $\varepsilon_{\mathrm{r}}$ is the dielectric constant of a vacuum, $\zeta$ is the zeta potential, and $\eta$ is the viscosity of the solution. The zeta potential was determined by measuring the electrophoretic mobility of the CNTs. Measurements were conducted 60 times for each sample to obtain mean zeta potentials.

A pure copper plate (JIS C1201P) with an exposed surface area of $10 \mathrm{~cm}^{2}\left(3.3 \times 3 \mathrm{~cm}^{2}\right)$ was used as the substrate. A copper plate containing phosphorus was used as the anode. A commercially available electrolytic cell (Model I, Yamamoto-Ms Co. Ltd) with internal dimensions of $6.5 \times 6.5 \times 9.5 \mathrm{~cm}^{3}$ was employed for electrodeposition, where the volume of the plating bath was $250 \mathrm{~cm}^{3}$. Electrodeposition was conducted under galvanostatic conditions $(0.1 \mathrm{~A})$ with aeration at $25^{\circ} \mathrm{C}$.

The surface and cross-sectional morphologies of the deposited films were examined using field emission-scanning electron microscopy (FE-SEM; SU-8000, Hitachi). A cross-section polisher 


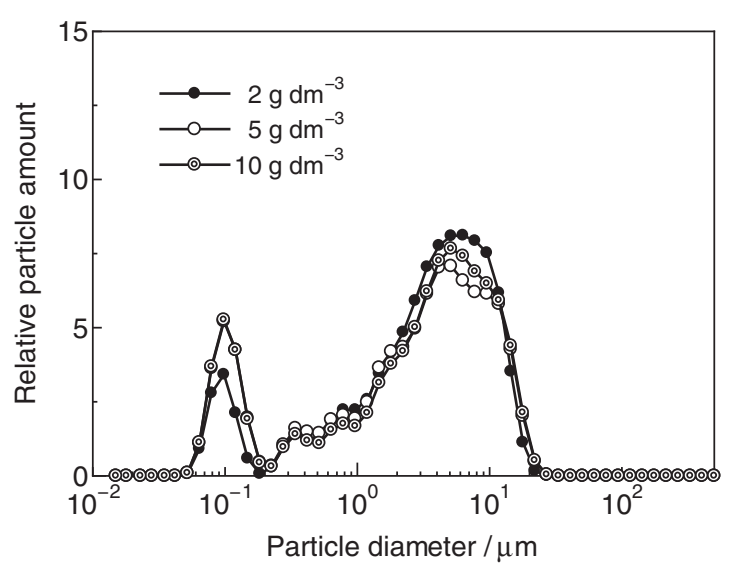

Figure 1. Particle size distribution for CNTs in composite plating baths with various $\mathrm{CNT}$ concentrations.

(SM-09010, JEOL) was used to prepare cross-sectional samples for FE-SEM observation. The CNT content in the films was evaluated by counting the number of CNTs in cross-sectional SEM images of the composite films. Two evaluation areas of $500 \mu \mathrm{m}^{2}$ were selected for each sample. Consequently, the CNT contents in the film were regulated as the CNT number per $1000 \mu \mathrm{m}^{2}$. Elemental mapping analysis of cross-sectional samples was conducted using electron probe microanalysis (EPMA; EPMA-1610, Shimadzu Seisakusho). The phase structures of the samples were examined using X-ray diffraction (XRD; XRD-6000, Shimadzu Seisakusho).

\section{Results and Discussion}

Figure 1 shows the dispersibility of CNTs in the plating baths with various concentrations of CNTs. Almost the same particle size distribution profiles were obtained for all CNT concentrations. Two large peaks are observed at around $100 \mathrm{~nm}$ and $10 \mu \mathrm{m}$ in each profile, which are similar to the diameter and the length of the single CNTs used, respectively. The particle shape was assumed to be spherical for the laser diffraction particle size analysis. However, the CNTs used have a rod-like shape and are not spherical; therefore, the particle diameter in the figure does not show the diameter of the CNTs in the strict sense of the word. However, these profiles clearly present the degree of dispersion of the CNTs, and the large two peaks at around $100 \mathrm{~nm}$ and $10 \mu \mathrm{m}$ correspond to the diameter and the length of a single CNT, respectively. ${ }^{27}$ Thus, it is considered that the CNTs are homogeneously dispersed as primary particles in the composite plating baths with CNT concentrations of $2-10 \mathrm{~g} \mathrm{dm}^{-3}$.

Figure 2 shows the zeta potentials of CNTs in the plating baths with various concentrations of CNTs. The zeta potentials of CNTs for all the CNT concentrations were relatively similar and small negative values $(-15$ to $-20 \mathrm{mV})$. Many investigations on the zeta potentials of CNTs in acidic or neutral aqueous solutions have reported small negative values. ${ }^{28-32}$ Furthermore, the zeta potentials of CNTs are generally affected by the CNT dispersant; ${ }^{33-36}$ anionic dispersants result in more negative zeta potentials and cationic dispersants yield more positive zeta potentials, while non-ionic dispersants do not have a strong effect on the zeta potential of CNTs. PA-5000 has carboxyl groups $(-\mathrm{COOH})$ and their acid dissociation is affected by $\mathrm{pH}$, i.e., carboxyl groups do not dissociate at lower $\mathrm{pH}$, but only at higher $\mathrm{pH}^{35}$ Therefore, PA-5000 should be an anionic dispersant at higher $\mathrm{pH}$ due to the acid dissociation of carboxyl groups $\left(-\mathrm{COO}^{-}\right)$. However, the $\mathrm{pH}$ of the plating bath used in this work was very low, so that PA-5000 should act as a non-ionic dispersant. This is one of the reasons for the small negative zeta potentials of CNTs in the plating bath, similar to those values reported for CNTs in acidic aqueous solutions without dispersants. ${ }^{21}$ The dispersibility of particles in the presence of dispersants is generally dominated by electrostatic and/or steric repulsion.

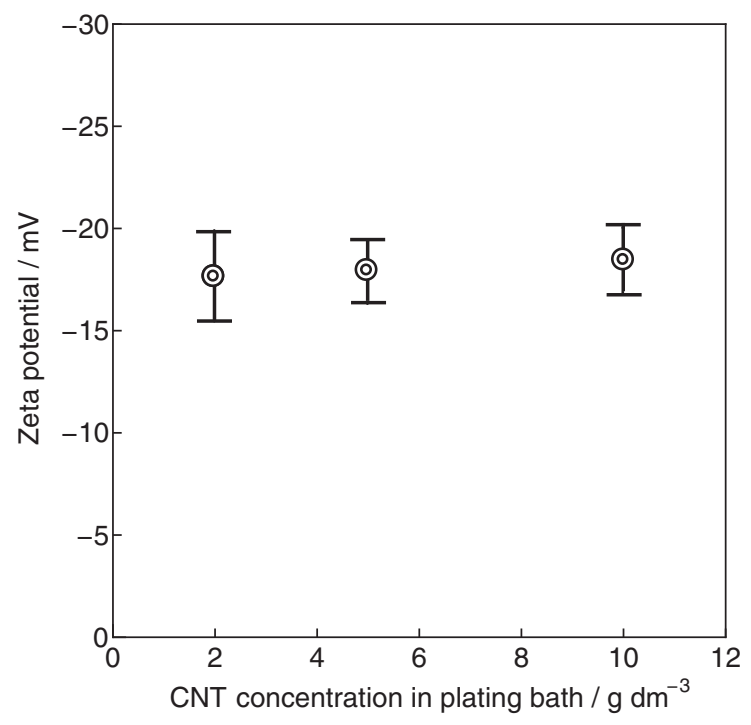

Figure 2. Zeta potentials for CNTs in composite plating baths with various CNT concentrations.

The electrostatic repulsion effect is related to the zeta potential. To stabilize dispersions by steric repulsion, absolute zeta potentials of at least $15 \mathrm{mV}^{33,34}$ and usually more than $25 \mathrm{mV}^{30,31,37}$ are required. In this study, the absolute values of the CNT zeta potentials were 15-20 mV; therefore, the dispersibility of the CNTs was considered to be dominated not only by electrostatic repulsion but also by the steric effects of PA-5000. On the other hand, the zeta potential of CNTs is expected to have an effect on the co-deposition process of CNTs with electrodeposited copper. During formation of the composite, the CNTs should adsorb onto the deposited copper surface and be incorporated in the deposited copper. The adsorption process includes both electrostatic adsorption and physical adsorption by Van der Waals force. The cathode has a more negative potential and the CNTs also have negative zeta potentials, so that co-deposition of the CNTs in the deposited copper should be inhibited by the electrostatic repulsion effect. However, the absolute zeta potential for the CNTs is not so large; therefore, the effect of electrostatic repulsion on the co-deposition process of CNTs with copper would not be significant.

Figure 3 shows surface SEM images of electrodeposits from the plating baths, both with $\left(10 \mathrm{~g} \mathrm{dm}^{-3}\right)$ and without CNTs, formed under various amounts of electric charge. For deposits formed using the plating bath without CNTs, very thin copper sheets (3DC1 films) with thicknesses in the range of ca. 30-40 nm were grown with a total thickness in proportion to the amount of electric charge (Figs. 3a-3d). For $300 \mathrm{C}$ of electric charge, the copper sheets appeared to be piled on each other (Fig. 3d). The gaps between the copper sheets increased with the amount of electric charge; therefore, the structural strength of the 3DC1 films decreased with an increase in the amount of electric charge. The piled structure is formed by the deformation of deposited copper sheets due to the pressure of aeration during electrodeposition and/or that of running water used for rinsing after the electrodeposition. For deposits formed from plating baths with CNTs, the CNTs were co-deposited in the thin copper sheets and the total thickness of the copper sheets was thicker with an increase in the amount of electric charge (Figs. $3 \mathrm{e}-3 \mathrm{~h}$ ). The CNTs in the deposits are distributed as primary particles. Thus, 3DC/CNT composite films were successfully fabricated by one-step electrodeposition. Compared to the 3DC1 film, the 3DC/CNT composite film consists of relatively thick copper sheets with thicknesses of ca. 70-80 nm. The total concentration of PA-5000 in both plating baths was the same $\left(3 \times 10^{-4} \mathrm{M}\right)$ and a portion of the PA-5000 must adsorb onto the CNTs as a dispersant in the composite plating bath; therefore, the free PA-5000 concentration in the 3DC/CNT composite plating bath must be lower than that in the $3 \mathrm{DC} 1$ plating bath, which could affect the thickness of the copper 

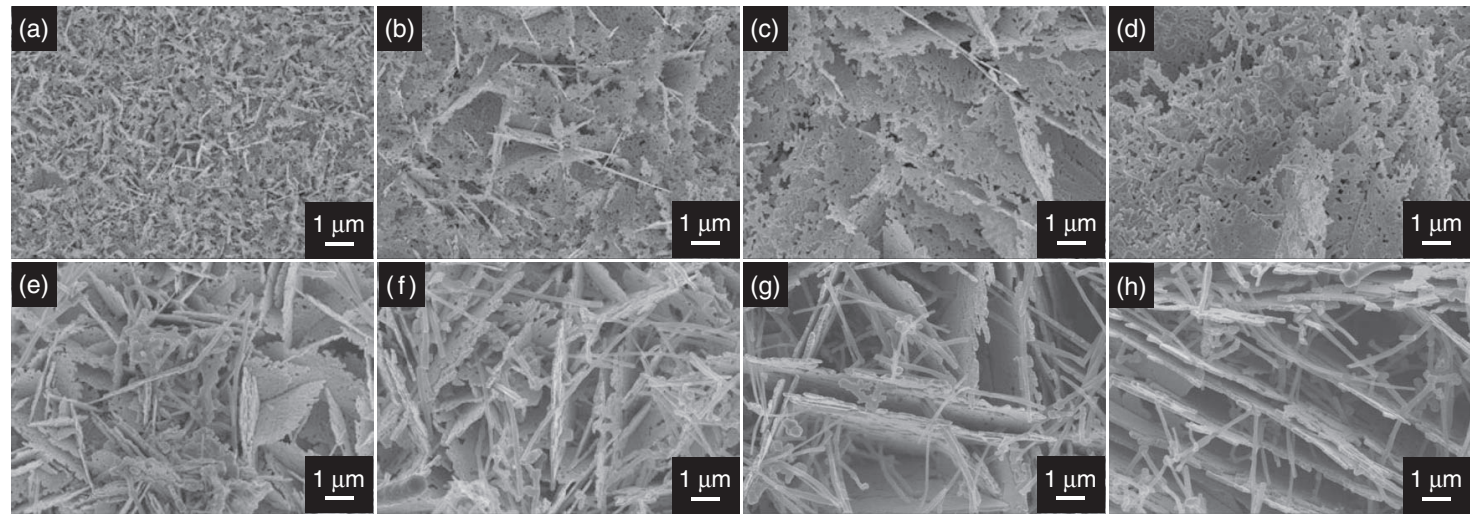

Figure 3. Surface SEM images of the $3 \mathrm{DC} 1$ and $3 \mathrm{DC} / \mathrm{CNT}$ films electrodeposited using various amounts of electric charge; $3 \mathrm{DC} 1$ with (a) $10 \mathrm{C}$, (b) $30 \mathrm{C}$, (c) 100 $\mathrm{C}$, and (d) $300 \mathrm{C}$, and 3DC/CNT with (e) $10 \mathrm{C}$, (f) $30 \mathrm{C}$, (g) $100 \mathrm{C}$, and (h) $300 \mathrm{C}$. The 3DC/CNT films were formed using a plating bath with a CNT concentration of $2 \mathrm{~g} \mathrm{dm}^{-3}$.

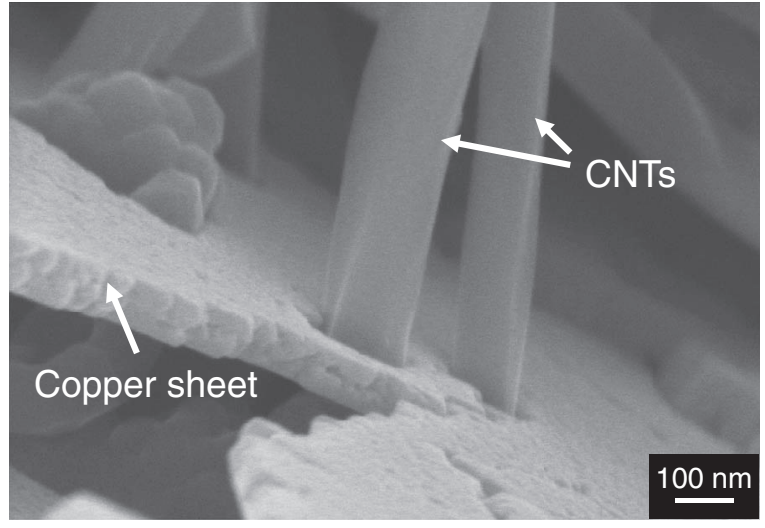

Figure 4. High magnification surface SEM image of the 3DC/CNT film electrodeposited at $300 \mathrm{C}$ using a plating bath with a CNT concentration of $2 \mathrm{~g}$ $\mathrm{dm}^{-3}$ (Fig. 3h).

sheets. The incorporation of CNTs into the 3DC/CNT composite film may also affect the thickness of the copper sheets. Figure 4 shows enlarged picture of Fig. 3h, where the CNTs penetrate though the copper sheet and are thus fixed in the deposited copper sheet.

Figure 5 shows a schematic illustration for the process whereby the CNTs are incorporated into the copper sheet. A copper sheet grows only in the planar direction while maintaining the same thickness (Fig. 5a). When the growing copper sheet meets a CNT (Fig. 5b), the copper sheet continues to grow without change in the growth direction and

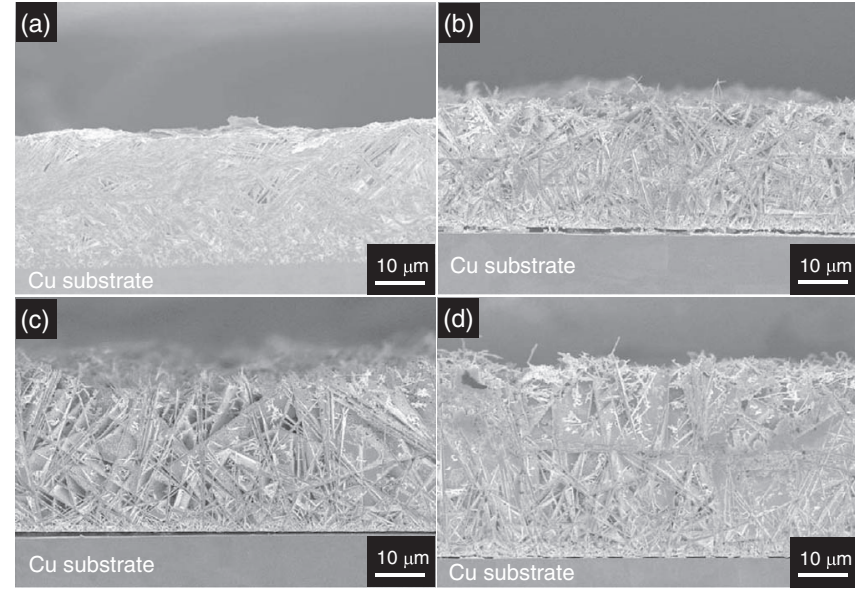

Figure 6. Cross-sectional SEM images of the 3DC/CNT composite films electrodeposited from plating baths with CNT concentrations of (a) $0 \mathrm{~g} \mathrm{dm}^{-3}$ (3DC1 film), (b) $2 \mathrm{~g} \mathrm{dm}^{-3}$, (c) $5 \mathrm{~g} \mathrm{dm}^{-3}$, and (d) $10 \mathrm{~g} \mathrm{dm}^{-3}$. The amount of electric charge for electrodeposition was $300 \mathrm{C}$.

while maintaining the thickness, which results in the incorporation of the CNT into the copper sheet (Fig. 5c).

Figure 6 shows cross-sectional SEM images of the 3DC1 film and the 3DC/CNT composite films fabricated from plating baths containing various CNT concentrations. All films were electrodeposited with $300 \mathrm{C}$ of electric charge. The thickness calculated for the compact

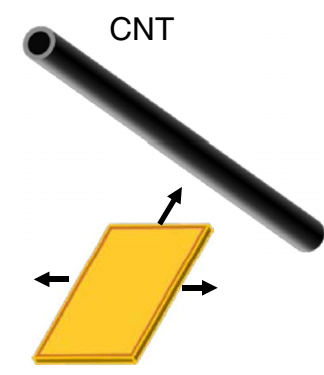

Copper sheet

(a)

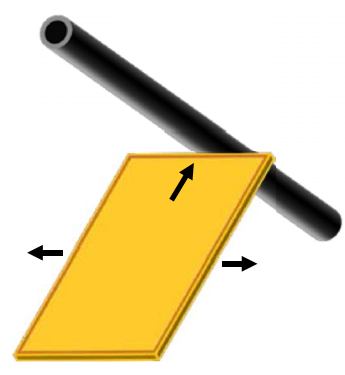

(b)

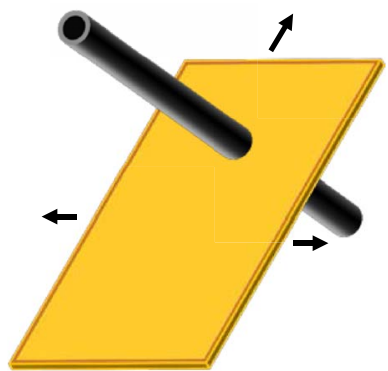

(c)

Figure 5. Schematic illustration of the process for CNT incorporation into the copper sheet. (a) A copper sheet grows only in the planar direction while maintaining the same thickness. A CNT is adsorbed onto other copper sheets near the growing copper sheet. (b) The growing copper sheet meets the CNT. (c) The copper sheet then continues to grow while maintaining the same thickness, which results in incorporation of the CNT into the copper sheet. 


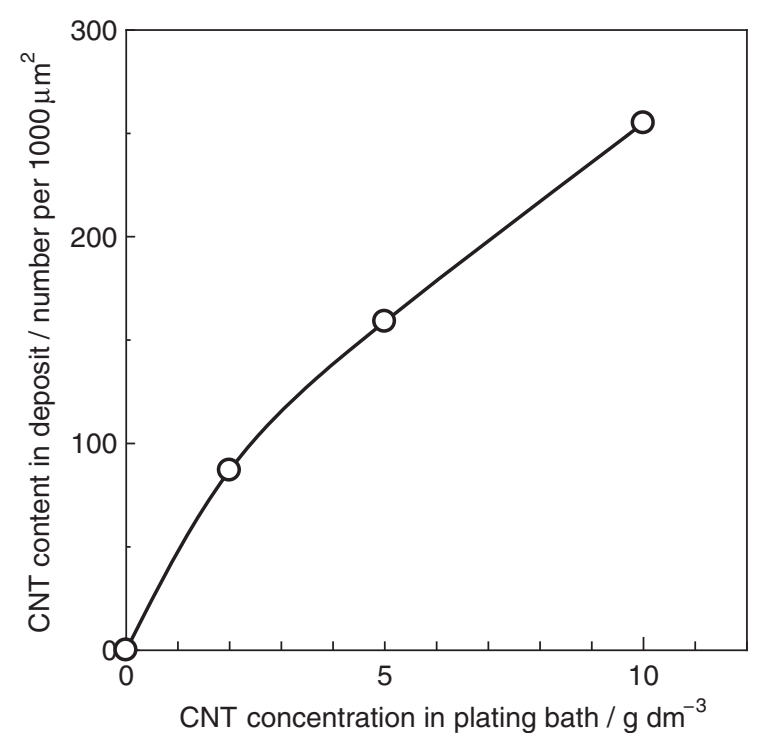

Figure 7. Relationship between CNT concentration in the plating bath and the CNT content in the electrodeposit.

copper film electrodeposited with $300 \mathrm{C}$ of electric charge is ca. 10 $\mu \mathrm{m}$. The observed film thickness of the 3DC1 film is ca. $28 \mu \mathrm{m}$ (Fig. $6 \mathrm{a}$; therefore, the porosity of the 3DC1 film is estimated to be ca. $64 \%$. Compared to the 3DC1 film, the film thicknesses (i.e., porosities) of the 3DC/CNT films are larger and the porosity increases with the CNT content in the plating baths. The estimated porosities were $66 \%, 71 \%$, and $74 \%$ for CNT plating bath concentrations of 2,5 , and $10 \mathrm{~g} \mathrm{dm}^{-3}$, respectively (Figs. $6 \mathrm{~b}-6 \mathrm{~d}$ ). The volume of the CNTs was ignored in the estimation of the porosity. The relationship between the $\mathrm{CNT}$ concentration in the plating bath and the CNT content in the de- posits is shown in Fig. 7. The CNT content was evaluated by counting the number of CNTs in cross-sectional SEM images of the composite films. Quantitative analysis of CNTs using energy-dispersive X-ray spectroscopy (EDS) and wavelength-dispersive X-ray spectroscopy (WDS) do not give accurate values for uneven samples such as the 3DC/CNT composite films. Direct weighing of the CNTs after removal of the copper matrix is one of the appropriate methods; however, the mass of CNTs was too small to weigh accurately in this study. The CNT content in the composite films increased with the CNT concentration in the plating bath, as shown in Fig. 7. Therefore, it is concluded that the porosity of the 3DC/CNT composite films increases with the CNT content in the film.

Figure 8 shows enlarged cross-sectional SEM images of those shown in Fig. 6. Figs. $8 \mathrm{a}-8 \mathrm{~d}$ and Figs. $8 \mathrm{a}-8 \mathrm{~d}^{\prime}$ show the upper and lower parts of Figs. $6 \mathrm{a}-6 \mathrm{~d}$, respectively. Fig. 8a shows the top region of the 3DC1 film. The copper sheets of the 3DC1 film are piled on each other, which results in a relatively packed morphology. This morphology corresponds to that of the surface morphology (Fig. 3d). These piles of copper sheets are formed by the deformation of deposited copper sheets due to the pressure of aeration during electrodeposition and/or that of running water during rinsing of the electrodeposited samples. In contrast, the piled texture is not observed in the 3DC/CNT composite films, and the interior spaces increase with the CNT content in the composite films (Figs. 8b-8d). Fig. 8a' shows the bottom region of the 3DC1 film. Although the copper sheets do not have a clearly piled texture as with the top region (Fig. 8a), the interior spaces between sheets are not so large. In contrast, similar to the top regions (Figs. $8 b-8 d$ ), the copper sheets at the bottom of the $3 \mathrm{DC} / \mathrm{CNT}$ composite films had larger interior spaces than the 3DC1 film (Fig. 8a'), and the interior spaces increased with the CNT content

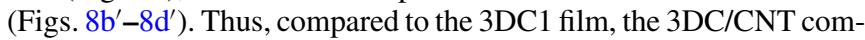
posite films have larger spaces inside the films and the interior spaces increase with the CNT content in the composite films. Figure 9 shows schematic diagrams of the 3DC1 film and 3DC/CNT composite film structures. The 3D structure is deformed when the thickness of the film increases, which results in the piled structure, i.e., a decrease of
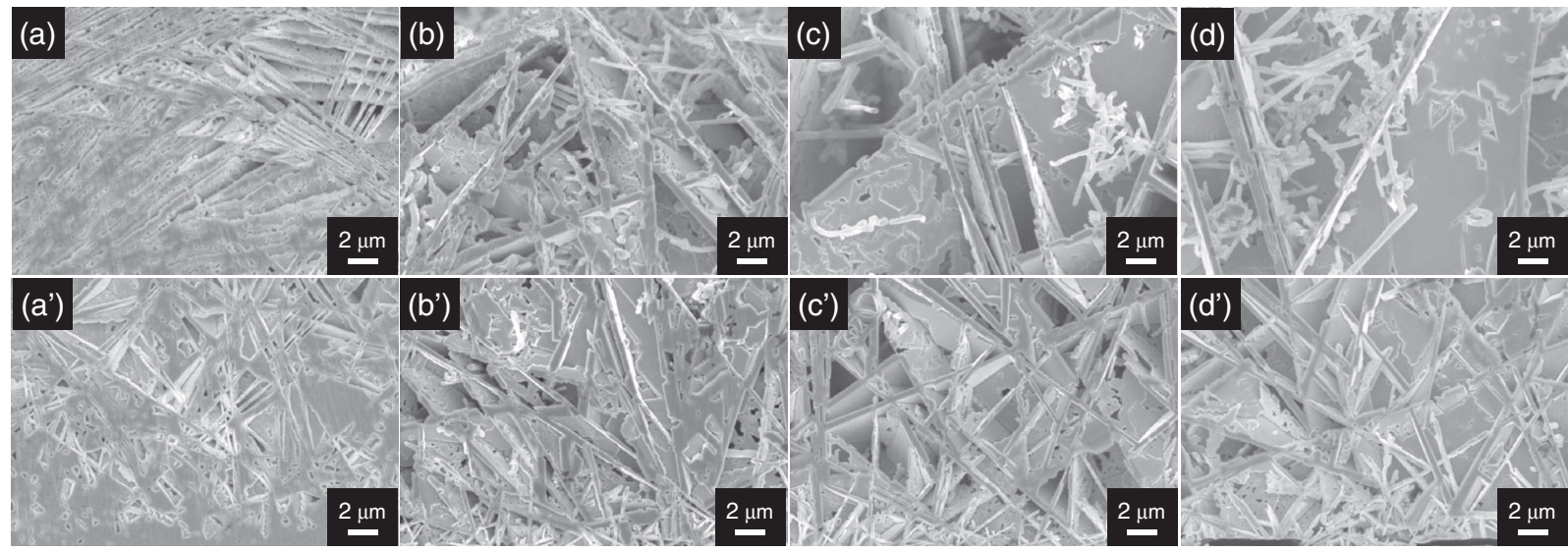

Figure 8. Enlarged SEM images of Fig. 6. (a) Upper and (a') bottom of Fig. 6a, (b) upper and (b') bottom of Fig. 6b, (c) upper and (c') bottom of Fig. 6c, and (d) upper and $\left(\mathrm{d}^{\prime}\right)$ bottom of Fig. $6 \mathrm{~d}$.

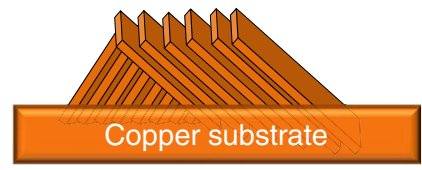

(a)

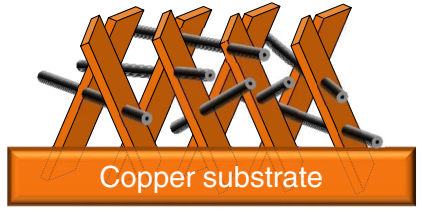

(b)

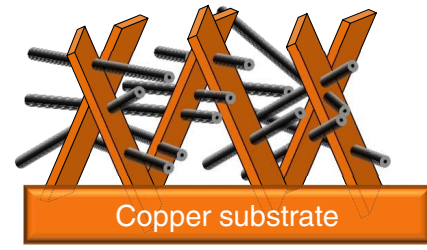

(c)

Figure 9. Schematic illustrations of 3D copper film structures for thick films. (a) 3DC1 film, (b) 3DC/CNT composite film with a small amount of CNTs, and (c) 3DC/CNT composite film with a large amount of CNTs. 

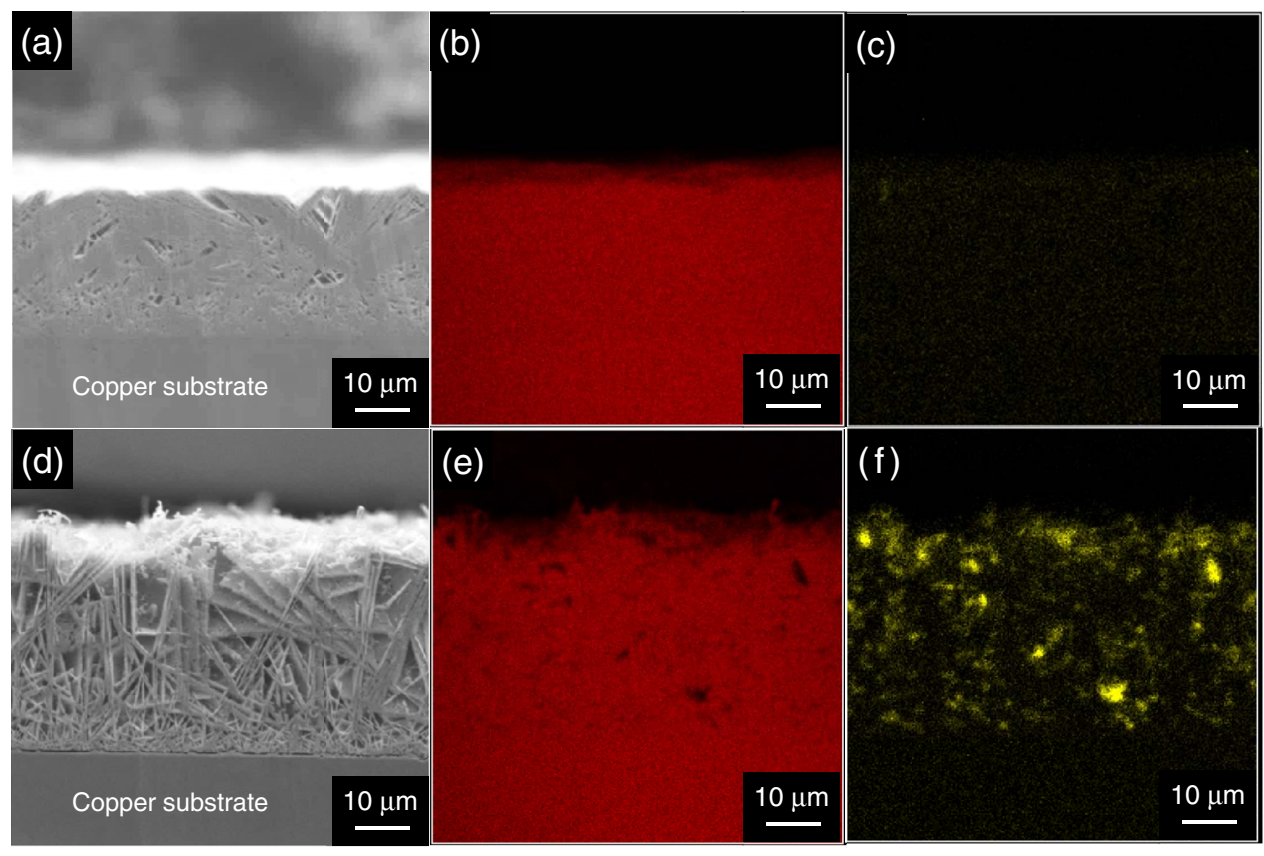

Figure 10. EPMA cross-sectional mapping analysis of the 3DC1 film and the 3DC/CNT composite film. (a) SEM image of the 3DC1 cross-section, and distributions of (b) copper and (c) carbon. (d) SEM image of the 3DC/CNT cross-section, and distributions of (e) copper and (f) carbon. The 3DC/CNT composite film was formed using a plating bath containing $10 \mathrm{~g} \mathrm{dm}^{-3}$ CNTs.

porosity (Fig. 9a). In contrast, the 3DC/CNT composite films maintain the 3D structure and have higher porosity than the 3DC1 film, even if the film thickness is increased, and the porosity increases with the CNT content in the film (Figs. 9b and 9c).

Figure 10 shows the results of EPMA elemental mapping analysis for cross-sections of the 3DC1 film and a 3DC/CNT composite film. The 3DC/CNT composite film was formed from a plating bath with a CNT concentration of $10 \mathrm{~g} \mathrm{dm}^{-3}$. For the $3 \mathrm{DC} 1 \mathrm{film}$, the copper is

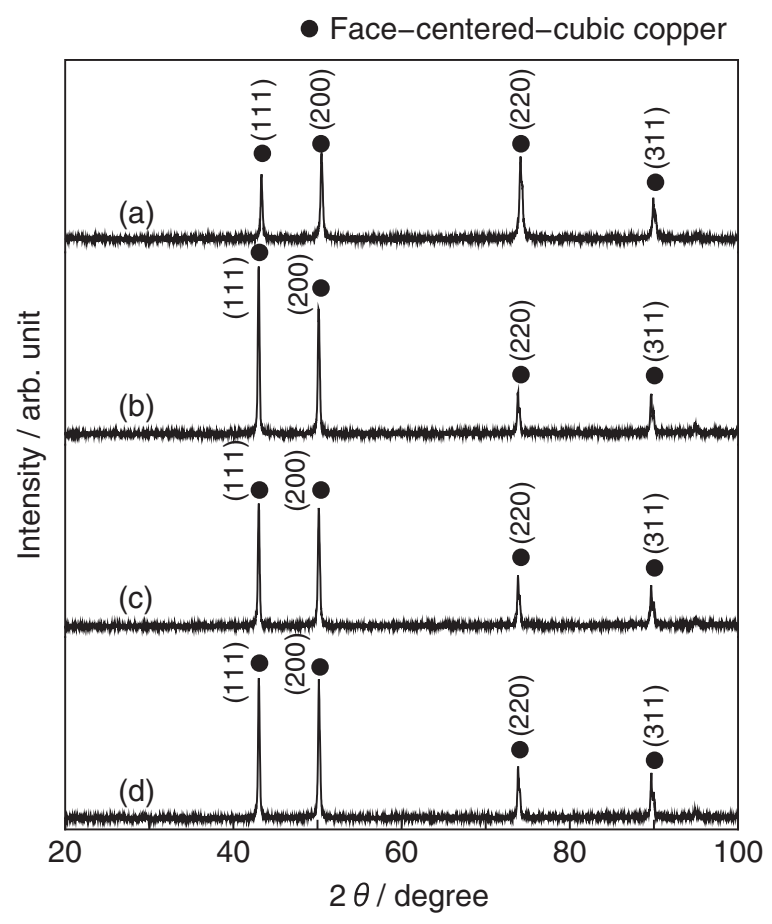

Figure 11. XRD patterns for (a) 3DC1, and for 3DC/CNT films formed from plating baths with CNT concentrations of (b) 2 , (c) 5 , and (d) $10 \mathrm{~g} \mathrm{dm}^{-3}$. distributed across the cross-section of the film and carbon is essentially absent (Figs. 10a-10c). In contrast, the 3DC/CNT composite film has relatively homogeneous distributions of carbon (CNTs) and copper across the cross-section.

Figure 11a shows an XRD pattern for the 3DC1 film and Figs. 11b $-11 \mathrm{~d}$ show those for the 3DC/CNT composite films electrodeposited from plating bath with various CNT concentrations. Every diffraction peak is sharp and assigned to the face-centered-cubic copper. No clear diffraction peak of the CNTs was observed in the XRD patterns, probably due to the small quantity of CNTs in the 3DC/CNT composite film, which would be insufficient for XRD detection. The results indicate that the 3DC/CNT composite films consist of relatively highcrystallinity copper sheets and CNTs.

The 3DC/CNT films fabricated in this study are expected to be used for various functional electrode applications, such as electrodes for electric double layer capacitors and lithium-ion batteries. ${ }^{38}$ The specific surface area and other properties of the 3DC/CNT composite films will be examined in future work.

\section{Conclusions}

A copper film with a $3 \mathrm{D}$ structure that consists of thin copper sheets and CNTs was fabricated by a one-step electrodeposition technique. The CNTs were incorporated tightly in the copper sheets and were distributed homogeneously in the composite film. This new 3D composite film is expected to be applied to various applications as a functional electrode material.

\section{Acknowledgment}

This work was supported by JSPS KAKENHI grant Number JP26289270.

\section{References}

1. S. Ghosh and O. Inganas, Adv. Mater., 11, 1214 (1999).

2. Y. Liu, S. Zha, and M. Liu, Adv. Mater, 16, 256 (2004).

3. D. R. Rolison and B. Dunn, J. Mater. Chem., 11, 963 (2001).

4. S. Zhang, Y. Xing, T. Jiang, Z. Du, F. Li, L. He, and W. Liu, J. Power Sources, 196, 6915 (2011)

5. H. C. Shin, J. Dong, and M. Liu, Adv. Mater., 15, 1610 (2003). 
6. H. C. Shin and M. Liu, Chem. Mater, 16, 5460 (2004).

7. T. Jiang, S. Zhang, X. Qiu, W. Zhu, and L. Chen, J. Power Sources, 166, 503 (2007).

8. J. Hassoun, S. Panero, P. Simon, P. L. Taberna, and B. Scrosati, Adv. Mater, 19, 1632 (2007).

9. S. Arai and T. Kitamura, ECS Electrochem. Lett., 3, D7 (2014).

10. S. Arai, M. Mendsaikhan, and K. Nishimura, J. Electrochem. Soc., 163, D54 (2016)

11. A. Oberlin, M. Endo, and T. Koyama, J. Cryst. Growth, 32, 335 (1976).

12. S. Iijima, Nature, 354, 56 (1991).

13. S. Rodney and C. L. Donald, Carbon, 33, 925 (1995).

14. J. P. Salvetat, J. M. Bonard, N. H. Thomson, A. J. Kulik, L. Forro, W. Benoit, and L. Zuppiroli, Appl. Phys. A, 69, 255 (1999).

15. M. F. Yu, B. S. Files, S. Arepalli, and R. S. Ruoff, Phys. Rev. Lett., 84, 5552 (2000).

16. S. Berber, Y. K. Kwon, and D. Tomanek, Phys. Rev. Lett., 84, 4613 (2000)

17. C. H. Yu, L. Shi, Z. Yao, D. Y. Li, and A. Majumdar, Nano Lett., 5, 1842 (2005).

18. T. Y. Choi, D. Poulikakos, J. Tharian, and U. Sennhauser, Nano Lett., 6, 1589 (2006).

19. H. Dai, E. W. Wong, and C. M. Lieber, Science, 272, 523 (1996).

20. T. W. Ebbesen, H. J. Lezec, H. Hiura, J. W. Bennett, H. F. Ghaemi, and T. Thio, Nature, 382, 54 (1996).

21. S. J. Yoo, S. H. Han, and W. J. Kim, Scripta Mater., 68, 711 (2013).

22. C. Arnaud, F. Lecouturier, D. Mesguichi, N. Ferreira, G. Chevallier, C. Estournes, A. Weibel, and C. Laurent, Carbon, 96, 212 (2016).
23. S. Arai, T. Saito, and M. Endo, J. Electrochem. Soc., 157, D147 (2010).

24. M. Endo, CHEMTECH, American Chemical Society, Sept., 568 (1998).

25. S. Arai and M. Endo, Electrochem. Commun., 5, 797 (2003).

26. S. Arai and M. Endo, Electrochem. Solid-State Lett., 7, C25 (2004).

27. K. C. Park, M. Fujishige, T. Takeuchi, S. Arai, S. Morimoto, and M. Endo, J. Phys Chem. Solids, 69, 2481 (2008).

28. M. Sano, J. Okumura, and S. Shinkai, Langmuir, 17, 7172 (2001).

29. L. Jiang, L. Gao, and J. Sun, J. Colloid Interface Sci., 260, 89 (2003).

30. L. Vaisman, G. Marom, and H. D. Wagner, Adv. Funct. Mater, 16, 357 (2006).

31. J. Lee, M. Kim, C. K. Hong, and S. E. Shin, Meas. Sci. Technol., 18, 3707 (2007).

32. E. F. de la Cruz, Y. Zheng, Erika Torres, W. Li, W. Song, and K. Burugapalli, Int. J. Electrochem. Sci., 7, 3577 (2012).

33. B. White, S. Banerjee, S. O'Brien, N. J. Turro, and I. P. Herman, J. Phys. Chem. C, 111, 13684 (2007)

34. Z. Sun, V. Nicolosi, D. Rickard, S. D. Bergin, D. Aherne, and J. N. Coleman, J. Phys. Chem. C., 112, 10692 (2008)

35. L. Chen and H. Xie, Thermochim. Acta, 506, 62 (2010)

36. S. Zhang, F. Lu, and L. Zheng, Colloid Polym. Sci., 289, 1815 (2011)

37. Y. T. Shieh, J. Y. Chen, Y. K. Twu, and W. J. Chen, Polym. Int., 61, 554 (2012).

38. S. Arai, M. Mendsaikhan, and K. Nishimura, J. Electrochem. Soc., 163 D54 (2016). 